\title{
METHOTREXATE AND END-STAGE KIDNEY DISEASE: A DANGEROUS COMBINATION
}

Marília Bulhões Calheiros ${ }^{1, \star}$, Camille Constanza Codogno Postigo Castro ${ }^{1}$, Aloma Guinami Scabora ${ }^{1}$, Vani Abreu de Souza Filho ${ }^{1}$, Alisson Aliel Vigano Pugliesi ${ }^{1}$, Manoel de Barros Bertolo ${ }^{1}$

1.Universidade Estadual de Campinas, Campinas (SP), Brazil.

*Corresponding author: lilabulhoesc@gmail.com

\section{BACKGROUND}

Methotrexate (MTX) is one of the main disease-modifying drugs used in rheumatology. However, adverse effects lead to discontinuation in $20 \%$ of the patients. One of the most severe effect is myelotoxicity due to its high gravity potential. One of the risk factors for this complication is chronic kidney disease, since the drug excretion is $90 \%$ renal. There are no clear indications about the safety of the drug in patients with chronic kidney disease or end stage kidney disease in renal replacement therapy. Even the use of low doses in these patients is controversial. Below, the report of an end-stage kidney disease patient on dialysis who - even with low doses of methotrexate - evolves with severe myelotoxicity and consequent fatal infectious complication.

\section{CASE REPORT}

A64-year-old female patient diagnosed with rheumatoid arthritis (RA) nine years ago. She had been on monotherapy with infliximab for five years with good disease control. The patient also had diagnoses of systemic arterial hypertension and stage IV chronic kidney disease. She evolved with dialysis urgency after an episode of acute pulmonary edema, definitively starting hemodialysis 3 times per week. The patient abandons RA treatment for about 6 months, returning to follow-up with high disease activity. Due to multiple infectious complications related to dialysis catheter, it was chosen not to restart biological therapy and initiate low doses of methotrexate $10 \mathrm{mg} /$ week plus folic acid. After 2 weeks of use, the patient develops nausea, vomiting, diarrhea, lesions in the oral mucosa and epistaxis. Laboratory tests showed severe pancytopenia: hemoglobin $7 \mathrm{~g} / \mathrm{dL}$, total leukocytes $140 / \mu \mathrm{L}$ and platelets 3000/ $\mathrm{LL}$. Hematopoietic growth factor, folinic acid and blood components transfusion were administered because of leukopenia and anemia, respectively; it was started daily hemodialysis and broad-spectrum antibiotic therapy. Despite all the intensive support, the patient died of refractory septic shock.

\section{CONCLUSION}

Patients with end-stage kidney disease are at higher risk of myelotoxicity with the use of methotrexate, even at low doses. Consideration should be given to the use of alternative drug classes for treatment in these patients. 